\title{
Lesão por armlock em atleta amador de jiu-jitsu: relato de caso
}

Armlock injury in amateur jiu-jitsu athlete: case report

Lesión de armlock en atleta amateur de jiu-jitsu: reporte de caso

Luis Guilherme Rosifini Alves REZENDE ${ }^{1}$

Filipe Jun SHIMAOKA ${ }^{1}$

Gunter Shin Iti Takamori SHIMABUKURO²

Luiz Garcia MANDARANO-FILHO ${ }^{1}$

Nilton MAZZER ${ }^{3}$

Cláudio Henrique BARBIERI ${ }^{3}$

${ }^{1}$ Médico Assistente da Cirurgião de Mão, Hospital das Clínicas da Faculdade de Medicina de Ribeirão Preto da Universidade de São Paulo, HC-FMRP-USP, Campus Monte Alegre, 14049-900 Ribeirão Preto - SP, Brasil

${ }^{2}$ Médico Assistente da Cirurgião de Mão, Hospital Estadual de Serrana do Complexo do Hospital das Clínicas da Faculdade de Medicina de Ribeirão Preto, Universidade de São Paulo, HC-FMRP-USP, 14150-000 Serrana - SP, Brasil

${ }^{3}$ Professor Titular e Chefe da Divisão de Cirurgia da Mão, Hospital das Clínicas da Faculdade de Medicina de Ribeirão Preto, Universidade de São Paulo, HC-FMRP-USP, Campus Monte Alegre 14049-910 Ribeirão Preto - SP, Brasil

\section{Resumo}

O jiu-jitsu brasileiro é uma modalidade de arte marcial que utiliza o princípio da imobilização para a execução de seus golpes. Este esporte tem apresentado aumento do número de praticantes nas últimas duas décadas. Isto implica no aumento do número de lesões, principalmente quando se considera o perfil dos atletas amadores. As lesões do cotovelo no jiu-jitsu estão entre as lesões mais comuns nesta modalidade esportiva. O mecanismo de trauma mais observado é a hiperextensão forçada durante a aplicação da chave de braço (armlock ou armbar). Apresentamos um relato de caso de um paciente de 26 anos com uma lesão aguda do complexo ligamentar medial por chave de braço durante uma luta de jiu-jitsu. O paciente foi submetido ao tratamento conservador, com imobilização e fortalecimento da musculatura flexo-pronadora. Realizou-se o retorno gradual ao esporte, apresentando bom resultado funcional do cotovelo e do membro superior após quatro anos de seguimento.

Descritores: Cotovelo; Ligamentos; Ferimentos e Lesões; Artes Marciais; Traumatismos em Atletas.

\section{Abstract}

Brazilian jiu-jitsu is a martial art modality that uses the immobilization principle to execute your strokes. This sport has shown an increase in the number of practitioners in the last two decades. This implies an increase in the number of injuries, especially when considering the profile of amateur athletes. Elbow injuries in jiu-jitsu are among the most common injuries in this sport. The most observed trauma mechanism is forced hyperextension during the application of the arm lock (armbar or armbar). We present a case report of a 26-year-old patient with an acute arm injury to the medial ligament complex during a jiu-jitsu fight. The patient was submitted to conservative treatment, with immobilization and strengthening of the flexopronator muscles. A gradual return to sport was performed, with good functional results of the elbow and upper limb after four years of follow-up.

Descriptors: Elbow; Ligaments; Wounds and Injuries; Martial Arts; Athletic Injuries.

\section{Resumen}

El jiu-jitsu brasileño es una modalidad de arte marcial que utiliza el principio de inmovilización para ejecutar tus golpes. Este deporte ha mostrado un incremento en el número de practicantes en las últimas dos décadas. Esto implica un aumento en el número de lesiones, especialmente si se considera el perfil de los deportistas aficionados. Las lesiones de codo en jiu-jitsu se encuentran entre las lesiones más comunes en este deporte. El mecanismo de trauma más observado es la hiperextensión forzada durante la aplicación del bloqueo del brazo (brazo o brazo). Presentamos un caso clínico de un paciente de 26 años con una lesión aguda en el brazo del complejo del ligamento medial durante una pelea de jiu-jitsu. El paciente fue sometido a tratamiento conservador, con inmovilización y fortalecimiento de los músculos flexo-pronadores. Se realizó una vuelta al deporte paulatina, con buenos resultados funcionales del codo y miembro superior a los cuatro años de seguimiento.

Descriptores: Codo; Ligamentos; Heridas y Traumatismos; Artes Marciales; Traumatismos en Atletas.

INTRODUÇÃO

O jiu-jitsu brasileiro é uma modalidade de arte marcial em ascensão nos últimos anos. Seus princípios técnicos promovem a imobilização do lutador através de golpes baseados em mecanismos de bloqueio articular, motivo pelo qual a modalidade também é conhecida como arte suave. Estes métodos de imobilização utilizam alavancas biomecânicas que potencializam a execução dos golpes para neutralizar o oponente. É um método eficaz, onde um indivíduo fisicamente mais fraco e menor é capaz de imobilizar um oponente muitas vezes maior e mais forte. Por este motivo, esta modalidade também tem sido amplamente utilizada para defesa pessoal ou lazer. Entretanto, mesmo fora do ambiente competitivo, a presença de lesões durante os treinamentos é um importante fator a ser considerado, tendo em vista os princípios utilizados para o esporte. Além disso, o esporte pode ser praticado em duas modalidades: com kimono ("Gi") e sem kimono ("No Gi") $)^{1-3}$. O esporte também é utilizado como um dos componentes do "mma" (mixed martial arts), que significa artes marciais mistas, onde o atleta utiliza os golpes do jiu-jitsu combinados aos golpes de modalidades de socos e chutes, como boxe tailandês.

Um dos principais golpes utilizados, já ensinado nas primeiras aulas de Jiu-Jitsu é a chave de braço, popularmente conhecida como armlock ou armbar. Neste golpe, realiza-se uma hiperextensão forçada do cotovelo, utilizando o quadril como fulcro, e o peso do corpo como alavanca (Figura 1). Este mecanismo promove estiramento das estruturas anteriores, associado ao impacto das estruturas posteriores, promovendo primeiramente a dor. Em competições, espera-se que o oponente "desista" ("tapout") quando há dor, significando que 0 golpe se encontra bem encaixado. Infelizmente, alguns atletas tentam resistir 
demais, ou o golpe é aplicado de forma muito rápida, ocasionando lesão das estruturas do cotovelo ${ }^{4,5}$.

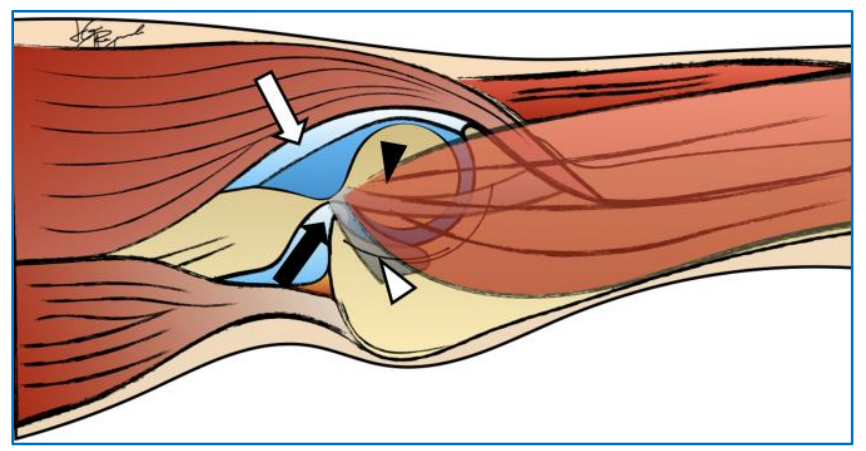

Figura 1: Mecanismos de trauma da chave de braço (armlock/armbar). Seta branca: cápsula anterior. Seta Preta: impacto posterior. Cabeça de seta branca: ligamento colateral medial. Cabeça de Seta Preta: origem da musculatura flexopronadora. (Fonte: elaborada pelo autor Luis Guilherme Rosifini Alves Rezende, 2021).

O objetivo deste estudo é apresentar um relato de caso de uma lesão por armlock em um atleta amador submetido ao tratamento conservador e o seu resultado funcional após quatro anos. Estudo realizado no Hospital das Clínicas da Faculdade de Medicina de Ribeirão Preto da Universidade de São Paulo, SP, Brasil.

\section{CASO CLÍNICO}

Paciente do sexo masculino, 26 anos de idade, destro, praticante de jiu-jitsu brasileiro há 3 anos, realizando treinos diários regularmente, em sessão de combate durante treinamento de rotina, quando foi imobilizado recebendo uma chave de braço em seu membro superior esquerdo, com o oponente em "guardamontada". O paciente referiu uma sensação de "rasgo" em seu cotovelo, associado a dor importante e dificuldade para apoiar o membro superior esquerdo no tatame para realizar a posição de "quatro apoios", e dificuldade em movimentos que dependessem de estresse em valgo ou extensão total do cotovelo. A luta foi interrompida e o paciente procurou auxílio médico imediato. Ao exame clínico, o paciente apresentava dor à palpação do epicôndilo medial do cotovelo esquerdo, delimitada até uma área de $2,5 \mathrm{~cm}$ distais ao epicôndilo. Não apresentava hematomas ou equimoses. Apresentou dor aos testes de estresse medial do cotovelo, com Milking Maneuver positivo para dor, impossibilitando a avaliação da instabilidade do cotovelo. $O$ paciente foi submetido à Ressonância Nuclear Magnética, cujos achados incluíram uma lesão da origem da massa flexo-pronadora, em relação ao epicôndilo medial, associada a uma lesão das bandas anterior e posterior do ligamento colateral medial. A presença de discreto edema posterior no cotovelo também foi observada, em conjunto com irregularidades na cápsula anterior (Figura 2).

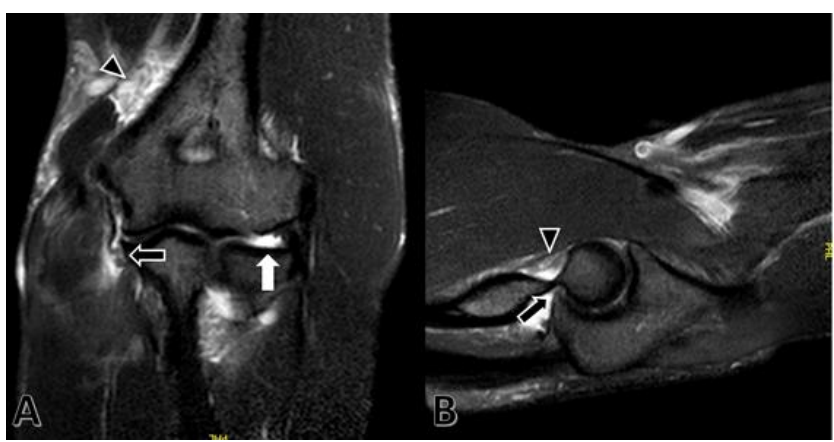

Figura 2: Alterações na ressonância magnética. A. Seta branca: derrame articular radiocapitelar. Seta preta: lesão do ligamento colateral medial. Cabeça de seta: lesão da origem da massa flexopronadora. B: Seta preta: impacto posterior. Cabeça de seta: irregularidade na capsula anterior.

Optou-se pelo tratamento conservador, com analgesia escalonada com Dipirona e Paracetamol com Codeína, associado ao uso de AINEs e Gelo e imobilização com órtese articulada durante 3 semanas. Neste período, o paciente realizou fisioterapia para flexo-extensão do cotovelo e prono-supinação do antebraço, de forma passiva e ativa. Após, foi iniciado um protocolo de fisioterapia ativa durante mais 3 semanas para flexo-extensão e prono-supinação com carga progressiva semanal. O paciente foi orientado a evitar movimentos que pudessem gerar estresse em valgo do cotovelo. Após 6 semanas, realizou-se - retorno para atividade física, através do exercício físico resistido, com um protocolo de fortalecimento para a musculatura flexo-pronadora, principalmente o flexor ulnar do carpo. Estes exercícios foram mantidos até 12 semanas. Após completar este período, realizou-se o retorno para o Jiu-Jitsu, para treinamento técnico e reeducação biomecânica e proprioceptiva do cotovelo. Aos 6 meses, o paciente prosseguiu com o seu processo de graduação no Jiu-Jitsu ("troca de faixa"), sem queixas de dores ou instabilidade residual. Observou-se alívio completo da dor, e, no período de quatro anos de seguimento, o paciente não apresentou alterações ou processos inflamatórios, como epicondilite medial. Em acompanhamento radiográfico após 4 anos observou-se formação de osteófito no compartimento medial (ulnoumeral) (Figura 3).

DISCUSSÃO

As lesões no cotovelo estão entre as lesões mais comuns no jiu-jitsu brasileiro, alcançando valores próximos a 19,4\% em alguns estudos. Estes valores são mais elevados em atletas menos graduados ou menos experientes, quando comparado aos atletas mais experientes ${ }^{2,3}$. 
Stephenson e Rossheim ${ }^{5}$ observaram que a maioria das lesões no jiu-jitsu ocorrem durante os treinamentos; porém, as lesões no membro superior tendiam a ser maiores durante as competições, alcançando valores de até $31 \%$ do total das lesões ${ }^{5}$. Petrisor et al. $^{6}$ também observaram uma maior taxa de lesões durante os treinamentos quando comparada a competição, sendo o membro superior um dos locais mais acometidos. Das Graças et al. $^{7}$ observou que o nível de graduação do atleta encontra-se relacionado ao risco de lesão no adulto. Já quando considerados os adolescentes, o sexo feminino e o tempo de exposição ao esporte eram os principais fatores de risco ${ }^{7}$.

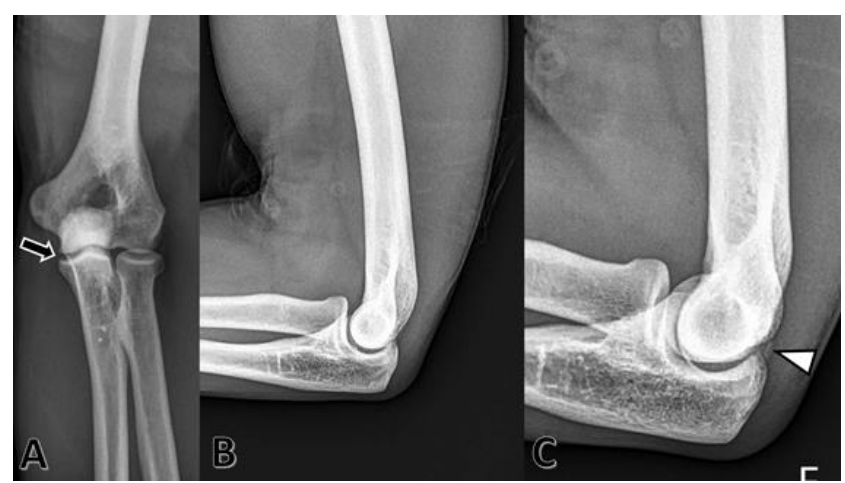

Figura 3: Radiografia em anteroposterior e perfil após 4 anos de lesão. A: anteroposterior, seta com formação de osteófito no compartimento medial (ulnoumeral). B: Perfil. C: visão obliqua, cabeça de seta mostrando osteófito posterior.

Almeida et al. ${ }^{4}$ apresentaram os padrões de lesões no cotovelo, secundários à chave de braço nos lutadores de jiu-jitsu. Eles apresentaram uma série de 5 casos, encontrando lesão do ligamento colateral medial ulnar e alteração da massa flexo-pronadora em todos os casos e $60 \%$ de alterações por impacto posterior úmero-olecraneano. Compactuando com as alterações observadas, nosso caso também apresentou as lesões apontadas por Almeida et al. ${ }^{4}$, com ruptura total da massa flexo-pronadora. Também encontramos alterações na cápsula anterior.

Scoggin et al. ${ }^{1}$ consideram as lesões no cotovelo como as mais comuns, sendo o mecanismo mais comum a lesão por armlock, em $71,4 \%$ dos casos. A lesão mais encontrada foi a lesão do ligamento colateral medial ulnar, e outras lesões incluem lesão do ligamento colateral lateral, lesões do bíceps e dor na região posterior do olecrano.

Acreditamos que as lesões no cotovelo ocasionadas por armlock ocorrem por estiramento das estruturas mais anteriores do cotovelo, associado ao impacto posterior entre a ponta do olecrano e úmero distal (Figura 3). Porém, acreditamos que tratamento cirúrgico deve ser proposto de acordo com a gravidade das lesões ligamentares do cotovelo. Neste caso, o paciente não possuía instabilidade do cotovelo, motivo pelo qual, optamos pelo tratamento conservador. Além disto, o fortalecimento da musculatura flexo-pronadora tem-se demonstrado eficaz no auxílio das lesões do ligamento colateral medial do cotovelo ${ }^{8,9}$.

Rettig et al. ${ }^{10}$ mostram ser possível o retorno ao esporte profissional em atletas de arremesso submetidos ao tratamento conservador. O mesmo foi observado para o basebol por Podesta et al. ${ }^{11} \mathrm{e}$, no basquetebol, por Ford et al. ${ }^{12}$. Ramos e Limpisvasti ${ }^{13}$ observaram que atletas não arremessadores também podem retornar ao esporte, porém, que poderá ser previsto através do padrão ligamentar de lesão observado na ressonância magnética. Apesar disto, não se conhece a evolução do padrão de lesões ligamentares do cotovelo em praticantes de jiu-jitsu, apesar da possibilidade da evolução para osteoartrite primária do cotovelo, comum em trabalhadores ou esportes com carga ${ }^{14}$. A literatura carece de estudos que nos mostrem os resultados do tratamento conservador ou a evolução das lesões ligamentares no cotovelo de atletas praticantes de jiu-jitsu.

CONSIDERAÇÕES FINAIS

Desta forma, conclui-se que a lesão ligamentar medial do cotovelo por chave de braço pode ser manejada com tratamento conservador, e que a avaliação da instabilidade ligamentar residual é uma condição fundamental para definição da modalidade terapêutica. Porém, mais estudos são necessários para definição do prognóstico das lesões ligamentares do cotovelo em atletas praticantes de jiu-jitsu.

\section{REFERÊNCIAS}

1. Scoggin JF 3rd, Brusovanik G, Izuka BH, Zandee van Rilland E, Geling O, Tokumura S. Assessment of Injuries During Brazilian JiuJitsu Competition. Orthop J Sports Med. 2014;2(2):2325967114522184.

2. Kreiswirth EM, Myer GD, Rauh MJ. Incidence of injury among male Brazilian jiujitsu fighters at the World Jiu-Jitsu No-Gi Championship 2009. J Athl Train. 2014;49(1):89-94.

3. Moriarty $C$, Charnoff J, Felix ER. Injury rate and pattern among Brazilian jiu-jitsu practitioners: A survey study. Phys Ther Sport. 2019;39:10713.

4. Almeida TBC, Dobashi ET, Nishimi AY, Almeida EB Junior, Pascarelli L, Rodrigues LMR. Analysis of the pattern and mechanism of 
elbow injuries related to armbar-type armlocks in jiu-jitsu fighters. Acta Ortop Bras. 2017;25(5):209-11.

5. Stephenson C, Rossheim ME. Brazilian Jiu Jitsu, Judo, and Mixed Martial Arts Injuries Presenting to United States Emergency Departments, 2008-2015. J Prim Prev. 2018;39(5):421-35.

6. Petrisor BA, Del Fabbro G, Madden K, Khan M, Joslin J, Bhandari M. Injury in Brazilian Jiu-Jitsu Training. Sports Health. 2019;11(5):432-39.

7. das Graças D, Nakamura L, Barbosa FSS, Martinez PF, Reis FA, Oliveira-Junior SA. Could current factors be associated with retrospective sports injuries in Brazilian jiujitsu? A cross-sectional study. BMC Sports Sci Med Rehabil. 2017;9:16.

8. Udall JH, Fitzpatrick MJ, McGarry MH, Leba $T B$, Lee TQ. Effects of flexor-pronator muscle loading on valgus stability of the elbow with an intact, stretched, and resected medial ulnar collateral ligament. J Shoulder Elbow Surg. 2009;18(5):773-78.

9. Park MC, Ahmad CS. Dynamic contributions of the flexor-pronator mass to elbow valgus stability. J Bone Joint Surg Am. 2004;86(10):2268-74.

10. Rettig AC, Sherrill C, Snead DS, Mendler JC, Mieling P. Nonoperative treatment of ulnar collateral ligament injuries in throwing athletes. Am J Sports Med. 2001;29(1):15-7.

11. Podesta L, Crow SA, Volkmer D, Bert T, Yocum LA. Treatment of partial ulnar collateral ligament tears in the elbow with platelet-rich plasma. Am J Sports Med. 2013;41(7):1689-94.

12. Ford GM, Genuario J, Kinkartz J, Githens T, Noonan T. Return-to-Play Outcomes in Professional Baseball Players After Medial Ulnar Collateral Ligament Injuries: Comparison of Operative Versus Nonoperative Treatment Based on Magnetic Resonance Imaging Findings. Am J Sports Med. 2016;44(3):723-28.

13. Ramos N, Limpisvasti O. UCL Injury in the Nonthrowing Athlete. Curr Rev Musculoskelet Med. 2019;12(4):527-33.

14. Wysocki RW, Cohen MS. Primary osteoarthritis and posttraumatic arthritis of the elbow. Hand Clin. 2011;27(2):131-37,

\section{CONFLITO DE INTERESSES}

Os autores declaram não haver conflitos de interesse

\section{AUTOR PARA CORRESPONDÊNCIA}

\section{Luis Guilherme Rosifini Alves Rezende}

HC-FMRP-USP Hospital das Clínicas da Faculdade de Medicina de Ribeirão Preto da Universidade de São Paulo - USP

Av. Bandeirantes, 3900 -

Campus Universitário Monte Alegre 14049-900 Ribeirão Preto, SP, Brasil.

E-mail: Igrezende@usp.br 